\title{
A SMOOTH SCISSORS CONGRUENCE PROBLEM
}

\author{
PETER GREENBERG
}

\begin{abstract}
Classifying space techniques are used to solve a smooth version of the classical scissors congruence problem.
\end{abstract}

\section{Introduction.}

1.1 The classical problem [8]. Let $B$ be the abelian group generated by the set of polygons in the plane, modulo the subgroup generated by elements $P-\Sigma P_{i}$, where $P \amalg P_{i}$ is a subdivision of a polygon $P$. Any subgroup $G$ of the group of affine motions of the plane acts on $B$. The problem is to compute the quotient group $H_{0}(G ; B)$ of $B$ by the subgroup generated by elements $g b-b$, with $g \in G, b \in B$.

$1.2 \mathrm{~A}$ smooth version. Our purpose is to state and solve a smooth version of the problem. Instead of polygons transforming under affine maps, we consider smooth curves transforming under diffeomorphisms.

The basic tool is a space $M(2.1)$ whose first singular integral homology group $H_{1} M$ is a smooth version of the group $B$. Diffeomorphisms of the plane act on $M$ and hence on $H_{1} M$. We employ a slight modification of a standard spectral sequence in our calculations.

1.3 Organization. $\S 2$ states the key definitions and results; the major proof is in $\S 3$. $\$ 4$ contains the proof of a lemma, and $\$ 5$ discusses the spectral sequence.

I would like to thank the referee for suggestions and for a simplification in the proof of Lemma 3.5.

2. Results. We require some definitions.

2.1 Definition. Let $M$ be the one-manifold of $C^{\infty}$ nonsingular curves in $\mathbf{R}^{2}$, defined as

$$
M=\amalg(a, b)_{f} / \sim
$$

where for each $C^{\infty}$ nonsingular embedding $f$ of an interval $(a, b)$ to $\mathbf{R}^{2}$ we take a copy $(a, b)_{f}$ of $(a, b)$, and where if $x \in(a, b)_{f}$ and $y \in(c, d)_{g}$ we set $x \sim y$ if and only if there exist neighborhoods $U$ of $x$ in $(a, b)_{f}$ and $V$ of $y$ in $(c, d)_{g}$ and a (not necessarily orientation preserving) diffeomorphism $h: U \rightarrow V$ such that $\left.f\right|_{U}=g \circ h$.

$M$ is a one-dimensional $C^{\infty}$ nonorientable non-Hausdorff manifold; let $i: M \rightarrow \mathbf{R}^{2}$ denote the obvious immersion. If $g: U \rightarrow V$ is a diffeomorphism between open sets in $\mathbf{R}^{2}$, let $i^{*} g: i^{-1} U \rightarrow i^{-1} V$ denote the resulting diffeomorphism between the open

Received by the editors August 20, 1982 and, in revised form, January 17, 1983.

1980 Mathematics Subject Classification. Primary 50D99, 58H05; Secondary 55H99.

Key words and phrases. Pseudogroup, classifying space. 
subsets $i^{-1} U$ and $i^{-1} V$ of $M$. Let $H_{1} M$ denote the first singular integral homology group of $M$.

2.2 Definition. Let $H_{0}\left(\Gamma^{\infty} ; H_{1} M\right)$ (resp. $H_{0}\left(\Gamma^{\Omega} ; H_{1} M\right)$ ) denote the quotient group of $H_{1} M$ by the subgroup generated by elements $\left(i^{*} g\right)_{*} b-b$, where $g: U \rightarrow V$ is an orientation preserving (resp. area and orientation preserving) $C^{\infty}$ diffeomorphism between open subsets of $\mathbf{R}^{2}$, and $b \in H_{1} M$ has support in $i^{-1} U$.

Our problem is to compute the groups just defined.

2.3 EXAMPLE. The Figure 8 curve (with orientation given by the arrow in Figure 1) defines an element of $H_{1} M$. Here is one demonstration that this element is 0 in $H_{0}\left(\Gamma^{\Omega} ; H_{1} M\right)$. The dotted curve indicates a part of $M$ used in each step.

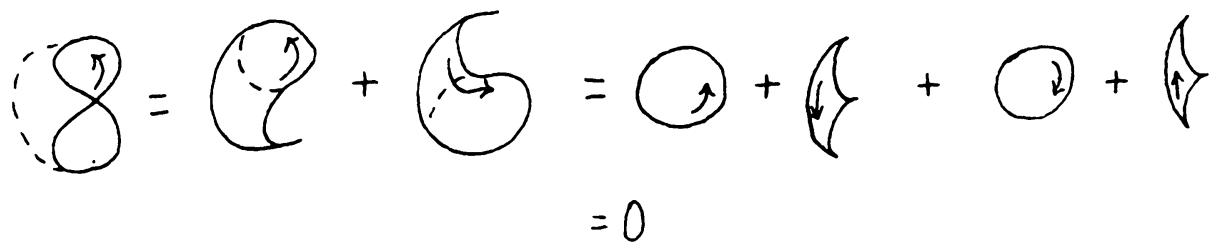

FIGURE 1

2.4 Definition. (i) The winding maps $W: H_{0}\left(\Gamma^{\infty} ; H_{1} M\right) \rightarrow \mathbf{Z}, W: H_{0}\left(\Gamma^{\Omega} ; H_{1} M\right)$ $\rightarrow \mathbf{Z}$. The tangent line field of $M$ defines a map from $M$ to $\mathbf{R} P^{1}$, and hence from $H_{1} M$ to $H_{1} \mathbf{R} P^{1}$. Picking an isomorphism of $H_{1} \mathbf{R} P^{1}$ with $\mathbf{Z}$ gives a map $H_{1} M \rightarrow \mathbf{Z}$, which pushes down to the maps $W$.

(ii) The area map $A$ : $H_{0}\left(\Gamma^{\Omega} ; H_{1} M\right) \rightarrow \mathbf{R}$ : If $b \in H_{1} M$, let $\underline{A}(b)=\int_{[b]} x d y$ (here [b] denotes the one-current of $\mathbf{R}^{2}$ associated to $\left.b\right) . \underline{A}(b)$ is the "algebraic area enclosed by $b$ ". $\underline{A}$ pushes down to the map $A$.

2.5 Theorem. The maps $W: H_{0}\left(\Gamma^{\infty} ; H_{1} M\right) \rightarrow \mathbf{Z}$ and $W \oplus A: H_{0}\left(\Gamma^{\Omega} ; H_{1} M\right) \rightarrow \mathbf{Z}$ $\oplus \mathbf{R}$ are isomorphisms.

2.6 Remark. We compare 2.5 with the classical result. Let $B$ (as in 1.1 ) be the abelian group generated by polygons in the plane, modulo the subgroup generated by subdivisions. Let $A G 1$ and $A S 1$ denote the group of orientation preserving affine maps of the plane and the subgroup of area and orientation preserving maps, respectively. Then $[8] H_{0}(A G 1 ; B)=0$, and area gives an isomorphism $A$ : $H_{0}(A S 1 ; B) \rightarrow \mathbf{R}$. There is no "winding map".

2.7 Remark. If in Definition 2.1 we glue the intervals $(a, b)_{f}$ together using orientation preserving diffeomorphisms $h$, we obtain a double cover $\tilde{M}$ of $M$, the one-manifold of $C^{\infty}$ oriented nonsingular curves in $\mathbf{R}^{2}$. There are winding maps $W$ : $H_{0}\left(\Gamma^{\Omega} ; H_{1} \tilde{M}\right) \rightarrow \mathbf{Z}$ and $W: H_{0}\left(\Gamma^{\infty} ; H_{1} \tilde{M}\right) \rightarrow \mathbf{Z}$ defined via the tangent unit vector map from $M$ to $S^{1}$, and an area map $A: H_{0}\left(\Gamma^{\Omega} ; H_{1} \tilde{M}\right) \rightarrow \mathbf{R}$. One can prove that $W$ : $H_{0}\left(\Gamma^{\infty} ; H_{1} \tilde{M}\right) \rightarrow \mathbf{Z}$ and $W \oplus A: H_{0}\left(\Gamma^{\Omega} ; H_{1} \tilde{M}\right) \rightarrow \mathbf{Z} \oplus \mathbf{R}$ are isomorphisms.

3. Proof of 2.5. We shall prove that $W: H_{0}\left(\Gamma^{\Omega} ; H_{1} M\right) \rightarrow \mathbf{Z} \oplus \mathbf{R}$ is an isomorphism. The proof for $W: H_{0}\left(\Gamma^{\infty} ; H_{1} M\right) \rightarrow \mathbf{Z}$ is almost identical (see Remark 3.6).

Recall that a topological category is a small category whose sets of objects and morphisms are topologized such that the structure maps of the category are 
continuous. The nerve of a topological category is a simplicial space; we use Segal's "thick" realization (denoted $\|\cdot\|$ in $[\mathbf{9}$, Appendix A]) to produce a classifying space functor $|\cdot|$ from topological categories to topological spaces.

3.1 Definition. Let $\Gamma^{\Omega}$ be the topological category whose space of objects is $\mathbf{R}^{2}$, and whose space of morphisms, denoted $\Gamma_{1}^{\Omega}$, is the space of germs of $C^{\infty}$ area and orientation preserving diffeomorphisms of $\mathbf{R}^{2}$, with the sheaf topology. Let $D, R$ : $\Gamma_{1}^{\Omega} \rightarrow \mathbf{R}^{2}$ denote the domain and range maps of $\Gamma^{\Omega}$.

The classifying space $\left|\Gamma^{\Omega}\right|$ is the "classifying space for $C^{\infty}$ codimension 2 foliation, with a transverse orientation and area form".

3.2 Definition. Let $\Gamma^{\Omega} \backslash M$ be the topological category of the action $\Gamma^{\Omega}$ on $M$; the space of objects of $\Gamma^{\Omega} \backslash M$ is $M$, and the space of morphisms $\left(\Gamma^{\Omega} \backslash M\right.$ ), of $\Gamma^{\Omega} \backslash M$ is the pullback:

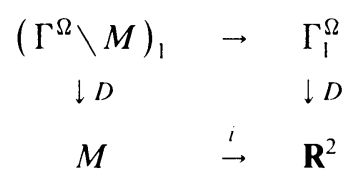

Let $i: \Gamma^{\Omega} \backslash M \rightarrow \Gamma^{\Omega}$ denote the continuous functor covering the map $i$.

Now we claim [2]

3.3 Proposition. There is a first quadrant spectral sequence $E_{p q}^{*}$, with differential $d^{n}$ of bidegree $(-n, n-1)$, which abuts to $H_{p+1}\left|\Gamma^{\Omega} \backslash M\right|$ and such that $E_{p 0}^{2}=H_{p}\left|\Gamma^{\Omega}\right|$ and $E_{01}^{2}=H_{0}\left(\Gamma^{\Omega} ; H_{1} M\right)$.

The spectral sequence is discussed in $\S 5$. To apply it to the proof of 2.5 we need two lemmas.

3.4 Lemma [4,2.6 AND 6, Lemma 1]. $H_{1}\left|\Gamma^{\Omega}\right|=0$ and $H_{2}\left|\Gamma^{\Omega}\right|=\mathbf{Z} \oplus \mathbf{R}$.

3.5 Lemma. $H_{1}\left|\Gamma^{\Omega} \backslash M\right|=\mathbf{Z} / 2$.

The proof of 3.5 is in $\S 4$.

Proof of Theorem 2.5. Let $K \oplus C: H_{2}\left|\Gamma^{\Omega}\right| \rightarrow \mathbf{Z} \oplus \mathbf{R}$ be the isomorphism of Lemma 3.4. Considering the spectral sequence 3.3, 2.5 will follow from the facts that $A \circ d^{2} \circ C^{-1}: \mathbf{R} \rightarrow \mathbf{R}$ is an isomorphism and that the image of $W \circ d^{2} \circ K^{-1}$ is $2 \mathbf{Z}$ (here $d^{2}$ is the differential for the $E^{2}$-term). These facts will follow from an explicit description of $d^{2}: H_{2}\left|\Gamma^{\Omega}\right| \rightarrow H_{0}\left(\Gamma^{\Omega} ; H_{1} M\right)$ for elements of $H_{2}\left|\Gamma^{\Omega}\right|$ represented by closed oriented two-manifolds with an area form.

Let $X$ be such a two-manifold, and let $[X] \in H_{2}\left|\Gamma^{\Omega}\right|$ be the corresponding homology class; $K[X]$ is the Euler characteristic of $X$, and $C[X]$ is the area of $X$. To describe $d^{2}[X]$, give a $C^{\infty}$ cell decomposition $X=\amalg \sigma_{i}$ of $X$ as in Figure 2. Each cell $\sigma_{i}$ can be mapped to $\mathbf{R}^{2}$ by an orientation and area preserving diffeomorphism $f_{i}$; the boundary of $f_{i} \sigma_{i}$, with orientation inherited from $X$, gives a cycle $\left[\partial f_{i} \sigma_{i}\right] \in H_{1} M$. Then $d^{2}[X]=\Sigma\left[\partial f_{i} \sigma_{i}\right]$ is well defined in $H_{0}\left(\Gamma^{\Omega} ; H_{1} M\right)$ and independent of the choice of $C^{\infty}$ cell decomposition of $X$. 

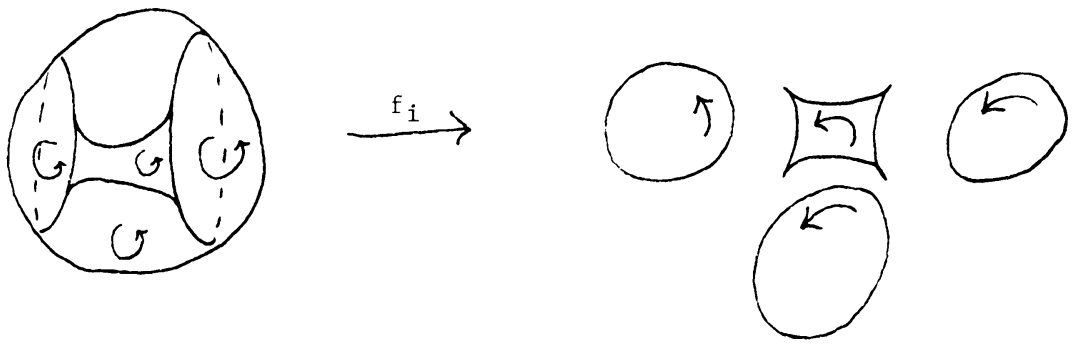

FIGURE 2

Clearly $A \circ d^{2} \circ C^{-1}$ is the identity, and a computation with $X=S^{2}$ shows that the image of $W \circ d^{2} \circ K^{-1}$ is $2 \mathbf{Z}$. This concludes the proof of 2.5.

3.6 REMARK. The proof that $W: H_{0}\left(\Gamma^{\infty} ; H_{1} M\right) \rightarrow \mathbf{Z}$ is an isomorphism follows $\S 3$, except for the substitution of the following lemma for Lemma 3.4.

3.7 Lemma [4, Theorem 3]. $H_{1}\left|\Gamma^{\infty}\right|=0$ and $H_{2}\left|\Gamma^{\infty}\right|=\mathbf{Z}$.

4. Proof of 3.5. The real line $\mathbf{R}$, embedded in $\mathbf{R}^{2}$ as the $x$-axis is a submanifold of $M$. Let $N$ be the discrete monoid of $\Gamma^{\Omega} \backslash M$-embeddings of the line; as a set

$$
N=\left\{s: \mathbf{R} \rightarrow\left(\Gamma^{\Omega} \backslash M\right)_{1} \mid D \circ s=\text { id and } R \circ s(\mathbf{R}) \subseteq \mathbf{R}\right\} .
$$

The translates of $\mathbf{R}$ by $\left(\Gamma^{\Omega} \backslash M\right)$, generate the topology of $M$, so by Theorem 1.2(ii) of [1] there is a weak homotopy equivalence $B N \rightarrow\left|\Gamma^{\Omega} \backslash M\right|$. Let us show that $\pi_{1} B N=\mathbf{Z} / 2$.

Let $K$ be the submonoid of $N$ consisting of elements which preserve the orientation of the line; it is not hard to see that the exact sequence $K \rightarrow N \rightarrow \mathbf{Z} / 2$ gives a homotopy fibration $B K \rightarrow B N \rightarrow B \mathbf{Z} / 2$. Since $\pi_{2} B \mathbf{Z} / 2=0,3.5$ will follow when we show that $\pi_{1} B K=0$.

So we show that the homomorphic image of $K$ in any group is trivial. Now $K$ is generated by elements $k$ which are the identity section in some open set $U$ (after [7], 3.1). But for any $U$ there is an $m \in K$ such that $m(\mathbf{R}) \subseteq U$; therefore $k m=m$ and $k$ must map to the identity of any group. So all of $K$ must map to the identity.

5. The spectral sequence 3.3. There is a spectral sequence for the action of a pseudogroup on a space, constructed in [2], which generalizes the spectral sequence for the action of a group on a space. The case at hand is an example of its application. We sketch the construction.

Let $C$ be the discrete category whose objects are contactible open subsets of $\mathbf{R}^{2}$, with morphisms area and orientation preserving embeddings between open sets. Note that (as in $[\mathbf{8}, \S 1])$ there is a weak homotopy equivalence between $|C|$ and $\left|\Gamma^{\Omega}\right|$.

Now recall the immersion $i: M \rightarrow \mathbf{R}^{2}$. Let $\underline{S}_{q}$ denote the complex of abelian group valued functors of $C$, where for $U$ an open subset of $\mathbf{R}^{2}, \underline{S}_{q} U=S_{q}\left(i^{-1} U\right)$, where $S_{q}$ is the usual singular $q$-chain functor. The spectral sequence for the complex $\underline{S}_{q}$ of functors satisfies 3.3. In particular, $E_{p 0}^{2}=H_{p}\left|\Gamma^{\Omega}\right|$ because $i^{-1} U$ is connected if $U$ is connected. 


\section{BIBLIOGRAPHY}

1. P. Greenberg, A model for groupoids of homeomorphisms, Thesis, M.I.T., Cambridge, Mass., 1982.

2. Extension and restriction for manifolds, preprint, 1982.

3. A. Haefliger, Feuilletages sur les varietes ouvertes, Topology 9 (1970), 183-194.

4. __ Homotopy and integrability, Manifolds-Amsterdam 1970, Lecture Notes in Math., vol. 197. Springer-Verlag, Berlin, 1971, pp. 133-163.

5. J. Mather, Integrability in codimension one, Comment Math. Helv. 48 (1973), 295-333.

6. D. McDuff, On groups of volume preserving diffeomorphisms and foliations with transverse form, Proc. London Math. Soc. 43 (1981), 295-320.

7. J. Palis and S. Smale, Structural stability theorems, Proc. Sympos. Pure Math., vol. 14, Amer. Math. Soc., Providence, R.I., pp. 223-231.

8. C.-H. Sah, Hilbert's third problem: scissors congruence, Pitman, London, 1979.

9. G. B. Segal, Categories and cohomologv theories, Topology 13 (1974), 293-312.

10. , Classifying spaces related to foliations, Topology 17 (1978), 367-382.

Department of Mathematical Sciences, North Dakota State University, Fargo, North DAKOTA 58105 